\title{
Laboratory Assessment of Kaolinite and Bentonite under Chemical- electrokinetic Treatment
}

\author{
Nasim Mosavat ${ }^{1 *}$, Erwin Oh ${ }^{1}$ and Gary Chai ${ }^{2}$ \\ ${ }^{1}$ Griffith School of Engineering, Griffith University, Australia \\ ${ }^{2}$ Centre for Infrastructure Engineering \& Management, Griffith University, Australia
}

\begin{abstract}
Electrokinetic treatment uses low intensity direct electric current or a low electric potential difference, to improve the engineering properties of subsurface soils while having minimum disturbance to the surface. This study aims to investigate the effects of chemical-electrokinetic treatment on properties of two types of clay soils at different intervals under the coupled Chemical, Hydraulic and Electrical (CHE) gradient. "Chemical-electrokinetic treatment" refers to improvement of soil with electrokinetic together with addition of chemical enhancement solution. The enhancement solutions used in this study are hydrated lime and saline solution. The combined effects of these processes together with various electrochemical and geochemical reactions would change the chemical composition of the soil porous medium, hence modifies the properties of the soil. The experiments were conducted in the laboratory using stainless steel electrodes under total applied voltage of $30 \mathrm{~V}$ for period of 15 days. Two types of fine-grained soils, kaolinite and bentonite, were used as they possess different mineralogy and geotechnical properties. The cumulative electro-osmotic flow and electric current flow through the soil samples were measured for period of 15 treatment days. Subsequently the specimens were tested for moisture content, $\mathrm{pH}$ and Atterberg Limit variations and the results have been reported. Test results revealed that there is a potential of developing this technique to improve engineering properties of finegrained soils, in particular to stabilise and improve soft soils for infrastructure management and constructions.
\end{abstract}

Keywords: Electrokinetic treatment; Chemical enhancement solution; Kaolinite; Bentonite

\section{Introduction}

The electrokinetic technology, which is an alternative method to the conventional ground improvement methods, involves electrochemical electro-osmosis stabilization in fine-grained soils. This method has been chosen in this study because it is cost-effective, applicable to insitu and ex-situ testings, rapid installation and easy to operate, having silent operation, having relatively short treatment duration, and can be implement in some existing structures. The use of the electrokinetic treatment, which is a comparatively new methodology, is being investigated in some parts of the world as a viable in situ soil stabilization and treatment method [1-11]. "Chemical-electrokinetic treatment" refers to improvement of soil with electrokinetic treatment together with the addition of chemical enhancement solution. The combinations of all complex electrochemical processes cause several modifications in physicochemical, hydrological and engineering properties of the fine-grained soils under an electric field for construction purposes. Therefore in this study, investigations are carried out at laboratory scale to identify the effects of chemical-electrokinetic processing on physical and chemical characteristics of two types of fine-grained soils. Moreover, this study aims to investigate the potential application of this technique as a viable in situ soil treatment method in particular to improve soil physical properties.

The principles of the electrokinetic treatment method involve applying a low Direct Current (DC) or a low potential gradient to electrodes inserted in the low permeable fine-grained soils. These soils possess specific mineralogical properties; hence they are electrically and chemically active. When a DC electric potential is applied to the soil, it stimulates the migration of electricity, pore fluid, ions and fine particles across the soil-liquid medium towards the oppositely charged electrode, hence creating a combined effects of a Chemical, Hydraulic and Electrical (CHE) gradient [12]. In this regard, the application of DC electric potential has various complex electrochemical effects such as:

i. The electrolysis reaction of water at the vicinity of electrodes results in an oxidation at the anode (generating an acid front) and reduction at the cathode (producing a base front) and that migrate towards each other [13]. The redox reactions are as follow:

$$
\begin{array}{ll}
\text { Anode: } 2 \mathrm{H}_{2} \mathrm{O}-4 \mathrm{e}^{-} \rightarrow \mathrm{O}_{2} \uparrow+4 \mathrm{H}^{+} & \mathrm{E}_{0}=-1.229 \\
\text { Cathode: } 2 \mathrm{H}_{2} \mathrm{O}+2 \mathrm{e}^{-} \rightarrow \mathrm{H}_{2} \uparrow+2 \mathrm{OH}^{-} & \mathrm{E}_{0}=-0.828
\end{array}
$$

Where $\mathrm{E}_{0}$ is the standard reduction electrochemical potential, which is the measure of the reactants in their standard states to proceed to products in their standard states.

ii. It establishes an electric potential difference, which may lead to generation of electro-osmosis [14]. Electro-osmosis is the flow of pore fluid through the soil that is recognized as the primary electrokinetic phenomena responsible for chemical species transport through the porous media. The flow rate depends on the coefficient of electro-osmotic permeability, $\mathrm{k}_{\mathrm{e}}[15,16]$.

iii. It generates the electric current flow mainly through the bulk pore fluid and along the particle surfaces through the Diffuse Double Layer (DDL) of the soil.

iv. It will lead to production of electrophoresis and electromigration through the sample. Electrophoresis is the motion of charged particles (usually micelles or colloids) relative to a fluid under an electric gradient, while electromigration is the gradual

*Corresponding author: Nasim Mosavat, Doctoral Research Fellow, Geotechnical Engineering, Griffith School of Engineering, Gold Coast campus, Griffith University, Australia, Tel: +61(0)755529057; E-mail: n.mosavat@griffith.edu.au

Received August 12, 2013; Accepted August 28, 2013; Published August 30 2013

Citation: Mosavat N, Oh E, Chai G (2013) Laboratory Assessment of Kaolinite and Bentonite under Chemical-electrokinetic Treatment. J Civil Environ Eng 3: 125 doi:10.4172/2165-784X.1000125

Copyright: (c) 2013 Mosavat N, et al. This is an open-access article distributed under the terms of the Creative Commons Attribution License, which permits unrestricted use, distribution, and reproduction in any medium, provided the original author and source are credited. 
movement of the ions or charged electrical species under an electric gradient

This technique can also be enhanced by introducing chemical compounds such as lime or calcium chloride to the soil at the appropriate electrode, preferably anode. The combined effects of these processes together with various geochemical reactions alter the chemical composition of the soil porous medium and thereby modify the physicochemical properties of the soil.

Although the technology has proved to be practical in many laboratory and field tests, complicated features such as many electrochemical reactions and soil-liquid interactions are still not fully understood. In other words, a fundamental understanding of complex micro-structural behavior of different clay minerals based on experimental investigations is limited. Field electro-osmotic mechanism is different from laboratory experiment and moreover, the chemical-electrokinetic treatment with the injection of lime and saline enhancement solutions (chemical stabilizers) has rarely been studied. Therefore there is a need for a detailed experimental program to be conducted for a better fundamental understanding of the physical and chemical applicability and efficiency of this technology. In this regard, this study aims to verify the use of the chemical-electrokinetic treatment as a viable in-situ soil treatment method in particular to improve two types of soft soils via an extensive laboratory experiments. This study will further discuss the effects of electrokinetic treatment on the conductance phenomena and index properties of kaolinite and bentonite subjected to hydrated lime and saline enhancement solutions using stainless steel electrodes under the total applied voltage of $30 \mathrm{~V} \mathrm{(1}$ $\mathrm{V} / \mathrm{cm}$ voltage gradient) for treatment duration of 15 days.

\section{Properties of soil samples}

According to the literature $[3,17]$, the electrokinetic treatment method is more effective on silty clays with moderate plasticity such as kaolinite. In addition, kaolinite displays much lower buffering capacity because of lower cation exchange capacity (CEC) compared with other clay minerals. Therefore in this study, kaolinite clay (S1) was initially used to perform the laboratory experiments. This was followed by the experiments conducted on a different type of soil, bentonite (S2). Bentonite can lead to serious engineering problems such as slope or wall failures, differential settlements and large cracks due to its expandable nature. Moreover, bentonite shows a much higher buffering capacity because of higher (CEC) compared with other clay minerals, such as kaolinite [18]. In this regard, bentonite was selected as an alternative experimental soil in order to compare the results of the chemical-electrokinetic treatment technique to stabilize and improve its engineering characteristics through an extensive laboratory program. The initial properties of soils S1 and S2 are summarized in Table 1.

\section{Experimental model and setup}

The laboratory experiments of the chemical-electrokinetic treatment of soil samples were carried out using the test model developed at the Griffith University. The schematic view of the experimental model has been shown in Figure 1. The chemical-electrokinetic tests are performed in clear glass rectangular tanks of $38 \mathrm{~cm}$ in length and 20 $\mathrm{cm}$ in width, under highly controlled laboratory conditions. The soil was homogenously mixed with tap water with relatively high moisture contents near the liquid limit value of the soil. For the electrokinetic technique to work, the soil moisture should be conductive and high enough to permit electromigration but not fully-saturated [19]. The experimental soil was then manually placed in the tank and compacted in three layers with a tamping rod in order to remove all visible air pockets so that the final compacted thickness was approximately 17.0 $\mathrm{cm}$. Tap water having a conductivity of $187 \mu \mathrm{S}$ at $25^{\circ} \mathrm{C}$ was used for the slurry preparation to resemble actual field application. Then, two hollow stainless steel tubes, possessing some drilled holes through their walls, to allow the chemical enhancement solutions to move freely into the sample, were placed $32 \mathrm{~cm}$ apart at the ends of the tank to be served as electrodes. An outlet from cathode chambers was connected to the vacuum pump to facilitate collecting cathode effluent and measuring the cumulative electro-osmotic flow volume. The power distribution cables were attached to the electrodes and connected to the DC power supply, providing total voltage of $30 \mathrm{~V}$.

The soils at various normalized distances of $0,0.25,0.5,0.75$ and 1 from the anode, were tested for variations in Moisture content (Mc), $\mathrm{pH}$ and Atterberg Limits as a result of chemical-electrokinetic process after duration of 15 days. The normalized distance is defined as the distance of the testing point from anode, over the total distance between the electrodes. In order to aid the electrokinetic process, soil samples were subjected to a slow continuous flow of two enhancement solutions (hydrated lime and saline solution). The saturated hydrated

\begin{tabular}{|c|c|c|c|}
\hline Soil characteristics & Soil S1 & Soil S2 & References/Methods \\
\hline Clay mineralogy & Kaolinite & Bentonite & - \\
\hline \multicolumn{4}{|l|}{ Atterberg limits } \\
\hline Liquid limit, LL (\%) & 43 & 650 & \multirow{4}{*}{ AS 1289.3.1.1-1995 } \\
\hline Plastic limit, PL (\%) & 38 & 200 & \\
\hline Plasticity index, PI (\%) & 6 & 450 & \\
\hline Linear shrinkage, LS (\%) & 4.9 & 27.2 & \\
\hline Activity & 0.15 & 4.9 & $\mathrm{PI} /$ Percentage of clay \\
\hline Specific gravity of solids, Gs & 2.6 & 2.8 & AS 1289.3.5.1-1995 \\
\hline Unified soil Classification & CL-ML & $\mathrm{CH}$ & ASTM D 2487-83 \\
\hline Textural classification & Silty clay & Heavy clay & Soil textural triangle by USDA ${ }^{1}$ \\
\hline Appearance (colour) & White & Light grey & - \\
\hline Percentage of clay $(\leq 0.002 \mathrm{~mm})(\%)$ & 38 & 92 & AS 1289.3.6.2-1995 \\
\hline Percentage of silt $(0.002-0.06 \mathrm{~mm})(\%)$ & 44 & 6 & AS 1289.3.6.2-1995 \\
\hline Percentage of sand $(0.06-2 \mathrm{~mm})(\%)$ & 18 & 2 & AS 1289.3.6.2-1995 \\
\hline Electrical conductivity of soil solution, EC ( $\mu S)$ & 591 & 397 & AS 1289.4.3.1-1997 \\
\hline $\mathrm{pH}$ & 8.6 & 10 & AS 1289.4.3.1-1997 \\
\hline
\end{tabular}

${ }^{1}$ United States Department of Agriculture 


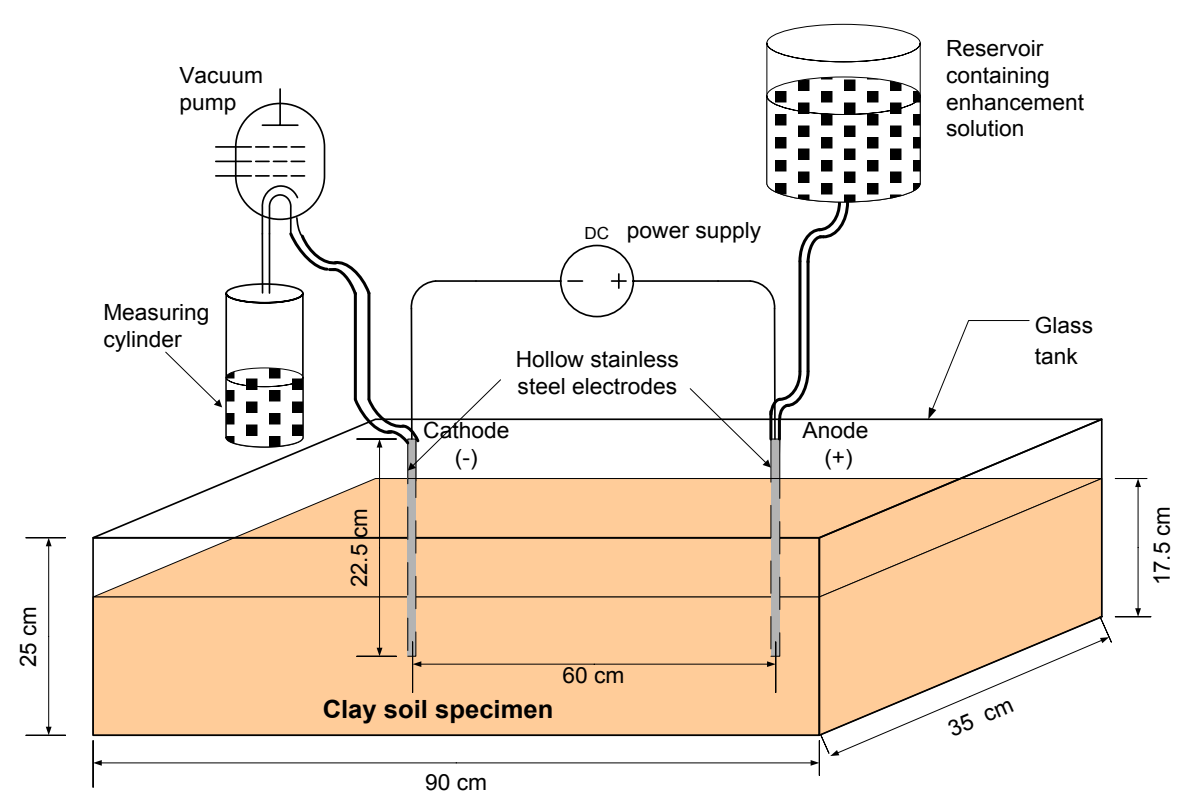

(a)

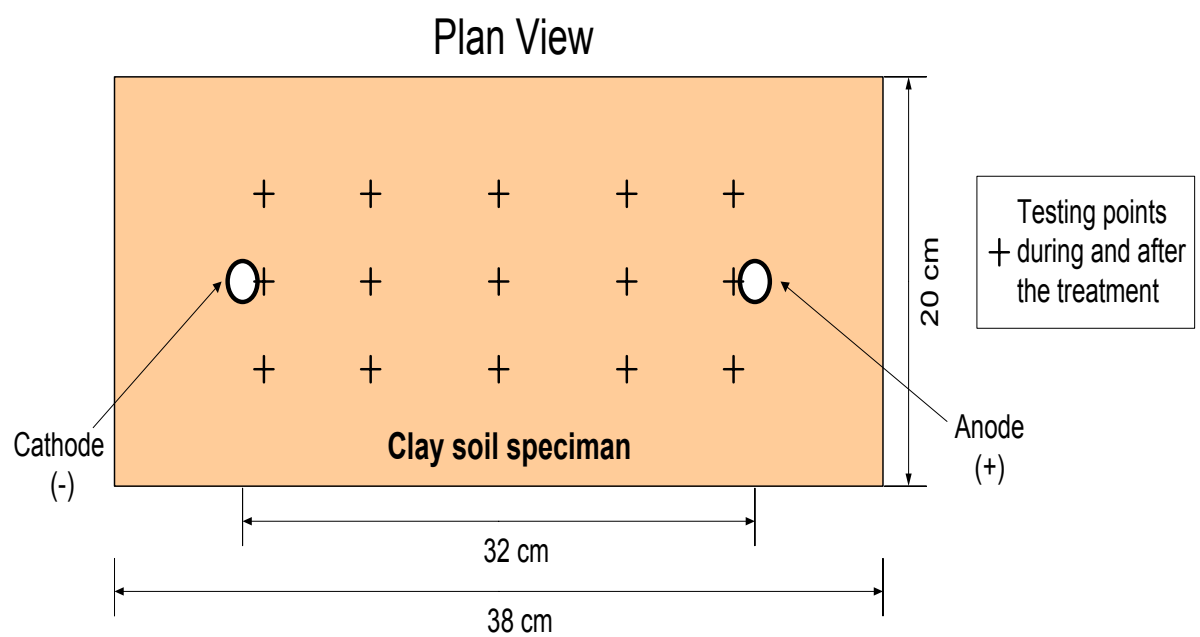

(b)

Figure 1: (a) Schematic view and (b) plan view of the laboratory set up for the electrokinetic treatment of the clay soil (not to scale)

\begin{tabular}{l|c|c}
\hline & Solution 1 & \multicolumn{1}{|c}{ Solution 2 } \\
\hline Properties & Hydrated lime & Calcium chloride $^{\mathrm{CaCl}_{2}}$ \\
\hline Molecular formula & $\mathrm{Ca}(\mathrm{OH})_{2}$ & 111 \\
Molar mass $(\mathrm{g} / \mathrm{mol})$ & 74.093 & 110.9848 \\
Exact mass & 73.968071 & $\mathrm{Na}_{2} \mathrm{SiO}_{3}$ \\
Appearance & Soft white powder & White powder \\
Odour & Odourless & Odourless \\
Toxicity & Low & Low to medium \\
Acidity & 12.06 & Thick liquid \\
Basicity & Odourless & Corrosive \\
\hline
\end{tabular}

Table 2: Properties of the enhancement agents used in this study.

lime in distilled water had $\mathrm{pH}$ value of 12.4 and electro-conductivity of $8.9 \mathrm{mS}$ and named as solution 1 . Similarly, $0.1 \mathrm{~N}$ calcium chloride was mixed with 1:100 sodium silicate solutions (by the volume of distilled water) and this saline solution was selected as second enhancement solution during the electrokinetic treatment, named as solution 2 . The $\mathrm{pH}$ of this solution was found to be 9.5 and electro-conductivity value of $15 \mathrm{mS}$. Sodium silicate $\left(\mathrm{Na}_{2} \mathrm{SiO}_{3}\right)$ is a thick liquid having higher viscosity compared with distilled water. It is an extremely water- 
soluble substance (liquid) that will turn water very viscous if enough is added. The physicochemical properties of the hydrated lime (slaked lime), calcium chloride and sodium silicate used in this study are shown in Table 2. The enhancement liquids were permitted through the soil via anode by use of a thin flexible PVC tube connected to an external plastic reservoir, at a small external hydraulic head (with very low discharge of solution, almost dripping). For testing, the upper 20 $\mathrm{mm}$ was first removed to ensure that any surface drying/wetting or disturbance effects were avoided.

\section{Test procedures}

The cathode effluent (liquid) was extracted mechanically via a vacuum pump from the cathode well to measure the accumulated electro-osmotic flow volume for period of 15 days. The electric current between the electrodes was manually monitored using a digital multimeter for everyday. The index property tests were performed on samples retrieved from regular sections having average normalized distances of $0,0.25,0.5,0.75$ and 1 from the anode, subjected to a constant applied voltage of $30 \mathrm{~V}$ with two different enhancement solutions, after 15 days of the chemical-electrokinetic treatment. The Moisture content $(\mathrm{Mc})$ and $\mathrm{pH}$ variations of the soil samples were evaluated by using the method described in Australian standard AS 1289.2.1.1-2005 and AS 1289.4.3.1-1997 respectively. The Atterberg Limits (Liquid Limit LL, Plastic Limit PL, linear shrinkage LS) were evaluated using the method described in the Australian Standard (AS 1289.3.1.1-1995).

\section{Results and Discussions}

\section{Conductance phenomena during chemical-electrokinetic process}

Upon the application of DC electric potential through the soil across the electrodes, the soil-liquid medium undergoes several physicochemical, hydrological and mechanical changes due to several complex electrochemical processes that take place within the soil porous media. Some of these processes are initiated immediately after the DC electric potential was applied (e.g. electrolysis of water, electroosmotic flow, current flow, etc), whereas several other progressions develop with time (e.g. variations in $\mathrm{Mc}, \mathrm{pH}$, etc). With the increase of treatment durations, some of these modifications (e.g. electrolysis of water, electro-osmotic flow and electric current flow) appear to continue but with a gradually decreasing rate. This is due to the several other system complications, which develop within the porous media hindering the continuation of chemical-electrokinetic processes. The most prominent observation of these experiments was the development of wet and dry areas between the electrodes due to positive electroosmotic flow from anode (positively charged electrode) to cathode (negatively charged electrode).

Cumulative electro-osmotic flow: With application of the chemical-electrokinetic treatment, simultaneous flows of fluid, electricity and other chemical species (e.g. $\mathrm{Ca}^{+2}$ ), take place under the combined effects of CHE gradient. In these experiments, within hours, liquids were observed accumulating in the cathode wells and were calculated as cumulative electro-osmotic flow through the sample. The Helmholtz-Smoluchowsky's (H-S) model is the most common theoretical electro-osmosis description and is widely accepted by geotechnical researchers $[16,18]$. This model is based on the assumption that fluid transport through the soil pores as a result of excess positive charge transport in the DDL towards the cathode as well as other chemical species [20]. The DDL consists of a relatively mobile layer (outer layer) and a fixed layer (inner layer). Under an applied electrical gradient, the mobile part of the DDL moves towards the negatively charged electrode, which imparts a viscous drag on the free water in the porous medium and thus advances the mobility of water. If the soil pores are treated as capillary tubes, then the coefficient of electro-osmotic permeability $\left(\mathrm{k}_{\mathrm{e}}\right)$ is expressed as:

$$
K_{e}=\frac{D \zeta}{\eta} n
$$

Where $\zeta$ is the zeta potential $(\mathrm{V}), \mathrm{n}$ is the porosity, $\eta$ is the viscosity $\left(\mathrm{FT} / \mathrm{L}^{2}\right)$ and $\mathrm{D}$ is the dielectric constant. The electro-osmotic flow rate is controlled by the coefficient of electro-osmotic permeability $\left(k_{e}\right)$, which is a measure of fluid flux per unit area of the soil per unit electric gradient. Therefore, according to the H-S model, the electro-osmosis flow rate $\left(q_{e o}\right)$ is then described by:

$$
q_{e o}=k_{e}\left(\frac{\Delta E}{\Delta L}\right) A
$$

Where $\Delta E$ is electric potential difference, $\Delta L$ is length of soil sample, and $\mathrm{A}$ is cross-sectional area of sample.

Based on the eq. (1), negative zeta potential (negative surface charge of the particles), will result in a positive electro-osmotic flow rate representing flow from anode to cathode, while positive surface charge cause electro-osmotic flow from cathode to anode. The transport of species across the porous media is mainly due to the electrokinetic transport mechanisms of electro-osmosis, electromigration and electrophoresis. Apart from these mechanisms, with the introduction of lime or saline solution, the long-term pozzolanic reactions may also take place at various degrees depending on the soil type (clay mineralogy), percentage of calcium, $\mathrm{pH}$ of the media and availability of water to facilitate pozzolanic reactions [12]. The variation of total liquid volumes in the cathode well for soils S1 and S2 due to electro-osmotic flow is presented in Figure 2. It can be seen from this Fig that higher electro-osmotic flow rate and consequently higher cumulative electroosmotic flow volume was recorded for soil S1 (kaolinite) in comparing with soil S2 (bentonite). However in both soils, with increasing processing time, the rate of electro-osmotic flow through the soil samples gradually decreased and the chemical-electrokinetic processes across the soil were mostly controlled by the ionic redistribution complications developed within the soils.

From Figure 2 it can also be observed that for both soils with hydrated lime enhancement, higher cumulative electro-osmotic flow volume has been recorded in comparing with saline enhancement. This can be due to the H-S theory, where the electro-osmosis permeability (eq 3 ) and electro-osmotic flow rate (eq 4) under an electrical gradient are indirectly proportional to the viscosity $(\eta)$ of the pore fluid. As mentioned previously, saline solution has higher viscosity compared to hydrated lime due to existence of water-soluble thick liquid; sodium silicate. Therefore, with saline enhancement the viscosity of the pore fluid increases and electro-osmotic flow rate decrease. This led to delaying of electro-osmotic flow through the soils and reduction of cumulated cathode effluent.

Electric current flow: Variation of electric current flow (I) over time across the soils S1 and S2 with hydrated lime and saline enhancement are presented in Figure 3. From this Figure it can be observed that all the tests begin with relatively high electric currents, while with passing processing time, the current reduces reaching almost constant values. Generally, as for the electrokinetic treatment with injection of enhancement solutions, in addition to the electrokinetic effects, the increase of cation in the soil will result in an increase of hydration of cations, which causes more absorbed water, along with the cation, 


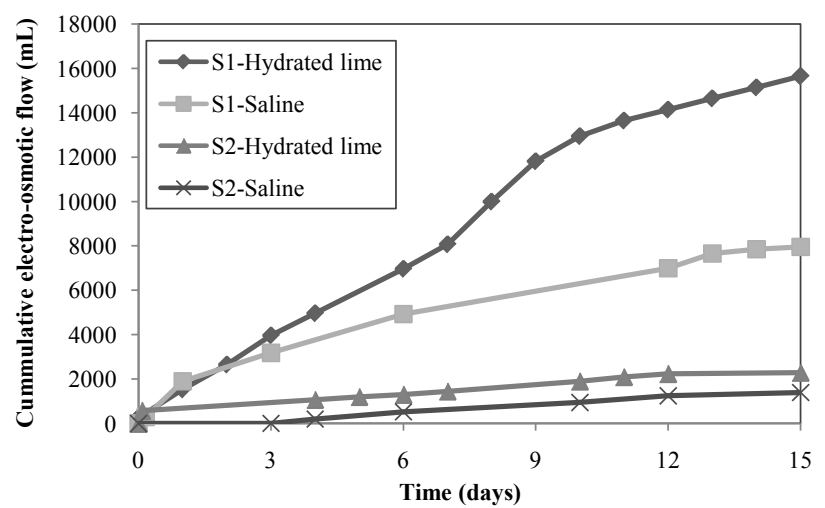

Figure 2: Variation of cumulative electro-osmotic flow volume over time for the soils S1 and S2 under chemical-electrokinetic treatment.

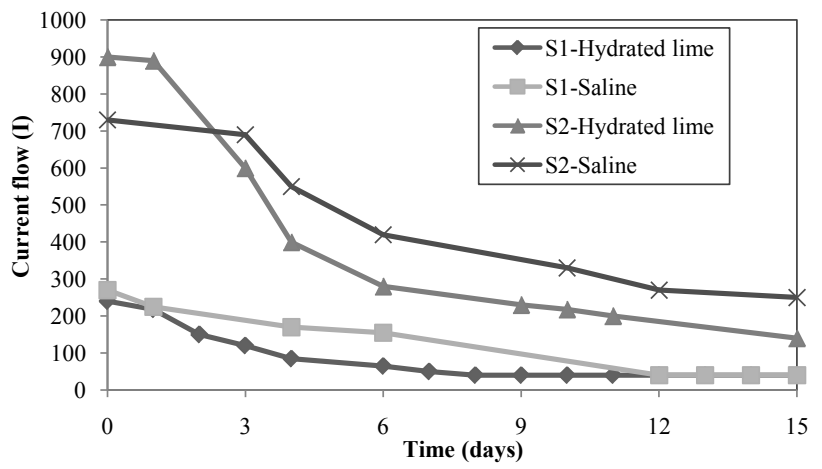

Figure 3: Variation of electric current flow (I) over time for the soils S1 and S2 under chemical-electrokinetic treatment.

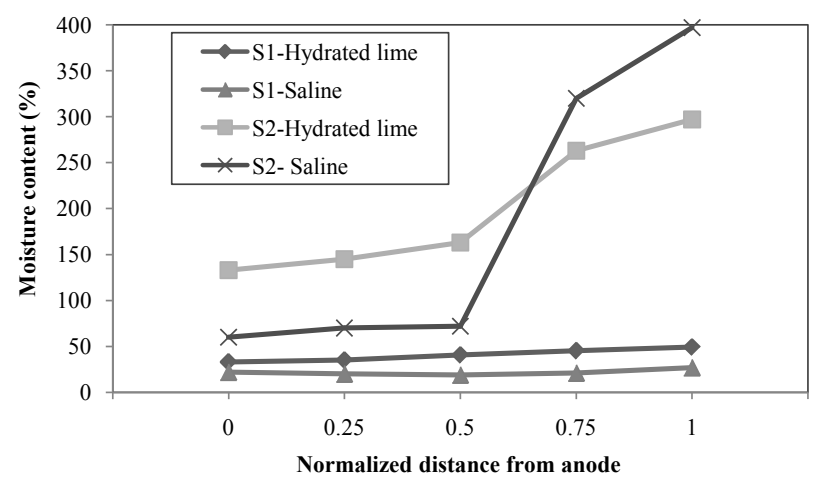

Figure 4: Variation of Mc of the soils S1 and S2 at regular normalized distances from the anode under chemical-electrokinetic treatment.

to migrate towards the cathode [4]. During the initial period of the chemical-electrokinetic treatment, a higher rate of electrolysis reactions (higher rate of $\mathrm{H}^{+}$production and higher ionic concentration) leads to a higher electro-conductivity of the pore solution and hence the total electric current flow through the sample will increase. However, with the passage of processing time, ionic concentration decreases due to diffusion and advection through the sample, a lower rate of electrochemical processes (electromigration) and probably adsorption to clay minerals, which causes lower ionic distribution across the sample and leads to lower electric current flow.
According to the Figure 3, soil S2 showed higher rate of electrolysis reactions and higher electric current flow through the soil sample. Moreover, the electric current flow through the soils seems to be higher with saline enhancement, which can be due to greater ionic concentration and conductivity of the initial pore solution. On the other hand, saline enhancement solution which was injected into the soils will increase electrochemical effects such as an increase of cation exchange and precipitation on the surface of clay particles leading to a higher current through the soil samples.

\section{Chemical-electrokinetic effect on index properties of the soils}

The chemical-electrokinetic process can modify the system chemistry of the porous media and therefore it can influence various index properties of the soils.

Chemical-electrokinetic effect on moisture content (MC) variations: variation of $\mathrm{Mc}$ of the soils $\mathrm{S} 1$ and $\mathrm{S} 2$ at regular normalized distances from the anode with hydrated lime and saline enhancement has been shown in Figure 4. Negative surface potential of the clay particles led to a positive electro-osmotic flow rate from the anode to cathode. This led to Mc variations across the tank, although the introduction of the enhancement solutions reduced the possibility of extreme dry regions at the anode. The results from the Figure 4 show that under the all chemical-electrokinetic processing conditions employed in this study, a considerable Mc gradient was built up in the kaolinite soil between the electrodes, dry regions near the anode and wet areas near the cathode. Soil S2 showed higher Mc values and variations due to possessing higher initial moisture content. The passage of enhancement solutions through the soil reduced the potential to form an extreme dry region near the anode. Similarly the existence of a vacuum pump at the cathode reduced the potential to form an extreme wet area near the cathode. The location of wet and dry regions depends on the properties of the soil such as mineralogical, permeability characteristics, clay type and content and DDL characteristics [21].

From Figure 4 it can also be observed that saline solution created lower Mc values in both soils especially near the anode. This can be due to the change in pore fluid characteristics such as viscosity of the saline enhancement solutions: calcium chloride and sodium silicate. As mentioned previously, sodium silicate is a thick liquid having a higher viscosity compared to distilled water. This higher viscosity of the pore fluid can lead to lower electro-osmotic and electrophoretic mobility through the soil samples. With the nature of high viscosity, saline solution would travel slower than hydrated lime solution. Further, bentonite has a natural property with high Atterberg Limits and clay

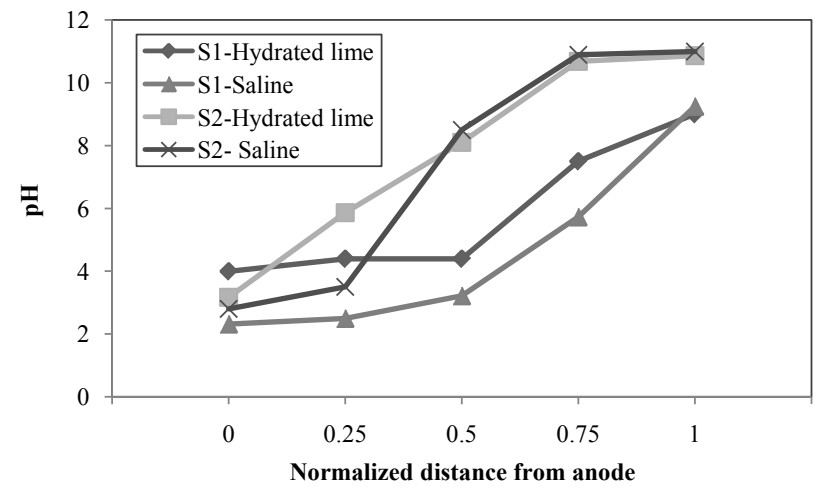

Figure 5: Variation of $\mathrm{pH}$ of the soils $\mathrm{S} 1$ and $\mathrm{S} 2$ at regular normalized distances from the anode under chemical-electrokinetic treatment. 
content (see Table 1). Therefore, bentonite with saline solution would exhibit high moisture content at the vicinity of the cathode

Chemical-electrokinetic effect on $\mathbf{p H}$ variations: The development of the $\mathrm{pH}$ gradient across the electrodes, for soils S1 and S2 measured at regular normalized distances from the anode with hydrated lime and saline enhancement is illustrated in Figure 5. As it can be observed from these results, it is noticed that under the all chemical-electrokinetic processing conditions employed in this study, a considerable $\mathrm{pH}$ gradient was being developed in both soils between the electrodes, forming an acid front at the anode and a base front at the cathode, although the passage of enhancement solutions through the soil reduced the extreme acidity near the anode. The $\mathrm{pH}$ measured for Soil S2 was found to be higher due to the higher $\mathrm{pH}$ value of soil S2 $(\mathrm{pH}=10)$ compared to Soil S1 $(\mathrm{pH}=8.6)$. The $\mathrm{pH}$ of the soil S2 remained unchanged during the early stages of the chemical-electrokinetic treatment due to the higher buffering capacity of bentonite, compared to kaolinite (soil S1). However with the passage of processing time, the $\mathrm{pH}$ of both soils started to drop reaching almost lowest value after 15 days of treatment.

From the results it can be also concluded that both enhancement solutions aimed to decrease the extreme acidity of the soil at the vicinity of the anode, especially with hydrated lime possessing higher $\mathrm{pH}$ value $(\mathrm{pH}=12.4)$ compared with saline solution $(\mathrm{pH}=9.5)$, hence possessing greater neutralizing potential. The presented results in Figure 5 also indicate that final acid-base chemistry ( $\mathrm{pH}$ profile) is almost identical (with $\mathrm{pH}$ values ranging from approximately $3 \pm 1$ at the anode and $9 \pm 1$ at the cathode under all test conditions) although the hydraulic conductivity, initial moisture content and fabric of both soils are different. Therefore it is apparent that the influencing factors are clay mineralogy, DDL characteristics and ionic concentration of the pore solution.

Chemical-electrokinetic effect on Atterberg limits variations: The plasticity properties of a soil depend on the soil moisture content as well as the variations in the chemistry of the soil-solution mixture. If a soil mineral has a greater ability to attract water to its surface, it can absorb more water before reaching the Liquid Limit (LL). That means more negative clay particle surfaces can absorb more water and result in higher LL values.

Variation of Atterberg Limits of the soils S1 and S2 at regular normalized distances from the anode with hydrated lime and saline enhancement are shown in Table 3. Results from the Atterberg Limit tests revealed that after 15 days of treatment, there was a net improvement in soil Atterberg Limits and plasticity characteristics in soil S1 from anode to cathode. However for soil S2 this trend was inverse being from cathode to anode. This can be due to the lower rate of electro-osmotic flow and electromigration in the soil S2 from anode through the cathode. From the test results it can be concluded that for soil S1, the soil-liquid interactions in the sample led to collapse of the DDL structure near the cathode and formation of 'flocs' which resulted in decrease of plasticity of the soil. As in many investigators' studies $[4,12], \mathrm{Ca}^{2+}$ released from the enhancement agents and migrating through the cathode can exchange $\mathrm{Na}^{+}$on the surface of clay, which makes the DDL of the clay thinner, leading to decrease of the Plasticity Index (PI) of the soil near the cathode.

For soil S2, the soil at the vicinity of the anode became flocculated and aggregated, leading to collapse of the DDL structure near the anode leading to low plasticity. This can be due to the lower rate of electromigration of the ions (e.g. $\mathrm{Ca}^{+2}$ ) from anode to cathode. Therefore towards the anode, the concentration of both exchangeable and water soluble $\mathrm{Na}^{+}$ions existing in bentonite is significantly reduced. This eventually creates a more flocculated clay structure and less negative clay particle surfaces leading to less plasticity. Conversely, closer to the cathode the $\mathrm{Na}+$ concentration is significantly higher due to accumulation of $\mathrm{Na}^{+}$ions under high $\mathrm{pH}$ environment, which leads to a more dispersed structure. Moreover, the shifting of $\mathrm{Na}^{+}$ions towards the cathode region would decrease the LL and PL of the soil at the vicinity of the anode. More $\mathrm{Na}^{+}$ions in the pore solution and on the particle surface increase the water sorption behavior of the soil since $\mathrm{Na}^{+}$ions can attract more water. The release of $\mathrm{Ca}^{+2}$ and $\mathrm{OH}^{-}$ions from the enhancement agents into the soil pore solution, subsequent cation exchange (substitution of $\mathrm{Ca}^{+2}$ ions for the existing $\mathrm{Na}+$ ions in Bentonite) and agglomeration-flocculation reactions, would suppress

\begin{tabular}{|c|c|c|c|c|c|c|}
\hline \multirow{2}{*}{ Soil type } & \multirow{2}{*}{ Anode enhancement solution } & \multirow{2}{*}{ Normalized distance from the anode } & \multicolumn{4}{|c|}{ Atterberg limits } \\
\hline & & & LL & PL & PI & LS \\
\hline \multirow{10}{*}{$\mathrm{S} 1$} & \multirow{5}{*}{ Hydrated lime } & 0 & 64 & 41 & 23 & 8 \\
\hline & & 0.25 & 56 & 39 & 17 & 7 \\
\hline & & 0.5 & 56 & 40 & 17 & 8 \\
\hline & & 0.75 & 56 & 41 & 17 & 6 \\
\hline & & 1 & 52 & 45 & 7 & 6 \\
\hline & \multirow{5}{*}{ Saline } & 0 & 80 & 44 & 36 & 12 \\
\hline & & 0.25 & 75 & 44 & 32 & 11 \\
\hline & & 0.5 & 76 & 45 & 31 & 10 \\
\hline & & 0.75 & 56 & 40 & 16 & 8 \\
\hline & & 1 & 55 & 41 & 14 & 8 \\
\hline \multirow{10}{*}{$\mathrm{S} 2$} & \multirow{5}{*}{ Hydrated lime } & 0 & 132 & 61 & 72 & 29 \\
\hline & & 0.25 & 132 & 71 & 62 & 33 \\
\hline & & 0.5 & 189 & 70 & 119 & 40 \\
\hline & & 0.75 & 398 & 89 & 308 & 54 \\
\hline & & 1 & 242 & 80 & 162 & 45 \\
\hline & \multirow{5}{*}{ Saline } & 0 & 96 & 58 & 38 & 29 \\
\hline & & 0.25 & 99 & 70 & 29 & 33 \\
\hline & & 0.5 & 219 & 80 & 139 & 42 \\
\hline & & 0.75 & 348 & 93 & 255 & 54 \\
\hline & & 1 & 201 & 88 & 113 & 45 \\
\hline
\end{tabular}

Table 3: Variation of Atterberg limits of the soils $S 1$ and $S 2$ at regular normalized distances from the anode under chemical-EK treatment. 
the thickness of the DDL and subsequently reduce the soil dispersion especially near the anode. Therefore for both soils, Liquid Limit (LL), Plastic Limit (PL), Plasticity Index (PI) And Linear Shrinkage (LS) of the soils appeared to be appropriate and sensitive parameters with which to determine whether chemical changes have occurred in the soil as a result of the chemical-electrokinetic treatment.

\section{Conclusions}

This paper has presented some laboratory results of a study undertaken to investigate the chemical-electrokinetic treatment effects in two types of clay soils (S1: kaolinite and S2: bentonite) subjected to two anode enhancement solutions under a predetermined voltage gradient of $30 \mathrm{~V}(1 \mathrm{~V} / \mathrm{cm})$ for duration of 15 days. From the analysis of the test results the following conclusions can be made:

With the application of DC electric potential through the sample, the soil-liquid medium undergoes several physical, chemical and electrical changes due to several complex electrochemical processes that take place within the soil porous media. The most prominent observation of these experiments was the development of wet and drier areas between the electrodes due to positive electro-osmotic flow from anode to cathode

Higher rate of electro-osmotic flow is observed during the early stage of the Chemical-electrokinetic process under both processing conditions while with processing time, a gradual decrease in the rate of electro-osmotic flow was noted. The rate of electro-osmotic permeability $\left(\mathrm{k}_{\mathrm{e}}\right)$ of soil $\mathrm{S} 1$ was found to be higher compared to soil S2 leading to higher cumulative electro-osmotic flow volume in the cathode.

The tests began with relatively high currents, however with processing time the current declined gradually. Soil S2 exhibited greater electric current flow. The electric current through the both soil samples found to be higher with saline enhancement.

A clear Mc gradient in both soils increasing from anode to cathode was built up in both soils. Saline enhancement led to lower electroosmotic flow and $\mathrm{Mc}$ variations due to higher viscosity of the pore fluid.

An obvious $\mathrm{pH}$ gradient was being developed in both soils between the electrodes, creating an acid front at the anode and a base front at the cathode, although the passage of anode enhancement solutions through the soil reduced the extreme acidity near the anode. This reduction in $\mathrm{pH}$ of the soil was higher with saline enhancement possessing lower neutralizing effect than hydrated lime.

During the chemical-electrokinetic treatment, there was a net improvement in Atterberg Limits and plasticity characteristics in soil S1 from anode to cathode and in soil S2 from cathode to anode. This can be due to the lower rate of electro-osmotic flow and electromigration in the soil S2 from anode through the cathode. In both soils the $\mathrm{Ca}^{2+}$ ion released from the enhancement agents could exchange $\mathrm{Na}^{+}$on the surface of clay, which makes the DDL of the clay thinner, leading to decrease of the PI of the soil near the electrode.

Based on the results presented, the index properties of the soils appeared to be appropriate and sensitive parameters with which to determine whether physicochemical changes have occurred in the soil as a result of the chemical-electrokinetic treatment. By concentrating on the factors of soil plasticity and drainage, the chemical-electrokinetic treatment method can be both economic and effective to stabilize and improve engineering properties of soft soils for constructions and infrastructure management. However, further investigations both in the lab and field are essential for the development of possible practical methods.

\section{References}

1. Acar YB, Alshawabkeh AN (1996) Electrokinetic remediation. I: Pilot-Scale Tests with Lead-Spiked Kaolinite. J Geotech Eng 122: 173-185.

2. Alshawabkeh AN, Acar YB (1994) Electrokinetic Remediation - Pilot-Scale Test-Results. Abstracts of Papers of the American Chemical Society 207: Envr 114

3. Pamukcu S, Wittle JK (1992) Electrokinetic Removal of Selected Heavy-Metals from Soil. Environ Prog 11: 241-250.

4. Ou CY, Chien SC, Wang YG (2009) On the enhancement of electro-osmotic soil improvement by the injection of saline solutions. Applied Clay Science 44 130-136.

5. Ivliev EA (2008) Electro-osmotic drainage and stabilization of soils. Soil Mechanics and Foundation Engineering 45: 211-218.

6. Ibanez JG, Singh MM, Pike RM, Szafran Z (1998) Laboratory experiments on electrochemical remediation of the environment Part 3: Microscale electrokinetic processing of soils. J Chem Educ 75: 634-635.

7. Grundl T, Reese C (1997) Laboratory study of electrokinetic effects in complex natural sediments. Journal of Hazardous Materials 55: 187-201.

8. De Battisti A, Ferro S (2006) Electrokinetic remediation: Methods of remediation of soils and ground waters (EREM 2005). Electrochimica Acta 52: 3345-3348.

9. Mitchell JK, Yeung AT (1990) Electro-kinetic flow barriers in compacted clay Transportation Res Rec 1-9.

10. Reddy KR, Ala PR (2006) Electrokinetic remediation of contaminated dredged sediment. Journal of ASTM International 6: 254-267.

11. Lee SW, Lo KY, Shang JQ, Micic S, Lee YN (2001) Electrokinetic strengthening of a marine sediment using intermittent current. Canadian Geotechnical Journal 38: 287-302.

12. Jayasekera $S$ (2008) An investigation into modification of the engineering properties of salt affected soils using electrokinetics. University of Ballarat, Australia.

13. Iyer R (2001) Electrokinetic Remediation. Particulate Science and Technology 19: $219-228$.

14. Acar YB, Gale RJ, Alshawabkeh AN, Marks RE, Puppala S, et al (1995) Electrokinetic remediation: Basics and technology status. J Hazardous Materials 40: 117-137.

15. Casagrande L (1949) Electro-osmosis in soils. Geotechnique 1: 159-177.

16. Mitchell JK (1993) Fundamentals of Soil Behaviour (2 ${ }^{\text {nd }}$ Edition). John Wiley \& Sons Inc, New York, USA.

17. Reddy KR, Saichek RE (2003) Effect of pH control at the anode for the electrokinetic removal of phenanthrene from kaolin soil. Chemosphere 51: 273287.

18. Acar YB, Alshawabkeh AN (1993) Principles of electrokinetic remediation Environ Sci Technol 27: 2638-2647.

19. Cauwenberghe LV (1997) Elektrokinetics: Technology overview report. Groundwater remediation technologies analysis center (GWRTAC), Pittsburgh, USA.

20. Alshawabkeh AN, Bricka RM (2001) Basics and application of electrokinetic remediation. Remediation Engineering of Contminated Soils, CRC Press, USA

21. Jayasekera S, Hall S (2007) Modification of the properties of salt affected soils using electrochemical treatments. Geotech Geol Eng 25: 1-10. 